\title{
Networks for the e-society
}

\author{
Michał Pióro • Jacek Rak • Krzysztof Szczypiorski
}

Published online: 1 September 2011

(c) The Author(s) 2011. This article is published with open access at Springerlink.com

\begin{abstract}
The 14th edition of International Telecommunications Network Strategy and Planning Symposium (shortly: Networks 2010) held in Warsaw, Poland. Networks 2010 was a four-day professional and scientific event organized by Warsaw University of Technology in co-operation with Polish Association of Telecommunication Engineers (SIT). The conference was focused on network design and planning methods including the issues of routing, traffic flows and optimization that are necessary to deliver the promise of the Network of the Future. Over 100 technical papers submitted by researchers form America, Asia, Australia, and Europe were carefully reviewed by the Members of the Technical Program Committee and external reviewers. The accepted 51 high quality papers were finally organized into 16 technical sessions. 6 keynote talks, 8 invited papers, and 4 tutorials enriched the conference program. Authors of the top 17 regular papers have been invited to publish extended versions of their papers in this special issue of Telecommunication Systems Journal.
\end{abstract}

The Networks 2010 conference (September 27-30, Warsaw, Poland) was the 14th event in the series of the International

\footnotetext{
M. Pióro · K. Szczypiorski $(\bowtie)$

Institute of Telecommunications, Warsaw University

of Technology, Nowowiejska 15/19, 00-665 Warsaw, Poland

e-mail: ksz@tele.pw.edu.pl

M. Pióro

e-mail: mpp@tele.pw.edu.pl

J. Rak

Gdansk University of Technology, Narutowicza 11/12, 80-233

Gdansk, Poland

e-mail: jrak@pg.gda.pl
}

Telecommunications Network Strategy and Planning Symposia. Since its first edition held in Paris thirty years ago, the meetings have been attracting world-class participants from all over the world, ranging from telecom operators, regulatory and standard bodies, through systems and software companies and research units from universities and industry, to systems integrators. The conference was organized by Warsaw University of Technology (Poland) in co-operation with Polish Association of Telecommunication Engineers (Poland). The technical sponsors of the conference were IEEE Poland Section, FP7 Network of Excellence Euro NF, and Polish Academy of Sciences.

Networks 2010 was a four-day professional and scientific event focused on network design and planning methods including the issues of network planning and optimization that are necessary to deliver the promise of the Network of the Future. The motto of Networks 2010 was Networks for the e-Society.

The conference presentations addressed mainly the issues of optical networks design, management and optimization techniques, quality of service, economic aspects of network design, routing in future generation networks, economic evaluation of new solutions, routing methods and algorithms (optimizing resource efficiency, robustness, and costs), evolution and future generation of wireless systems, optimization in network planning including resilience, and network security.

Around 100 technical papers submitted by researchers from America, Asia, Australia, and Europe were carefully reviewed by the Members of the Technical Program Committee and external reviewers. The accepted 51 high quality papers were finally organized into 16 technical sessions. On top of that, 6 keynote talks, 8 invited papers, and 4 tutorials enriched the conference program. The conference was attended by more than 130 participants. Conference papers 
were published in printed proceedings and are also available at IEEE Xplore.

Authors of the top 17 regular papers have been invited to publish extended versions of their papers in this special issue of Telecommunication Systems Journal.

In "Volume-oriented routing and its modifications", Mateusz Żotkiewicz and Walid Ben-Ameur present a new routing paradigm where each traffic demand is routed independently of the other demands: the volume-oriented routing. The paper by David Hock, Matthias Hartmann, Michael Menth, Michał Pióro, Artur Tomaszewski and Cezary Żukowski entitled "Comparison of IP-Based and Explicit Paths for One-to-One Fast Reroute in MPLS Networks" propose a mixed-integer model for optimizing the path layout for explicitly calculated paths, which can either produce single paths and route entire traffic along those paths, or generate multiple paths and spread the traffic among those paths providing load balancing. In "Optimizing Network Load Balancing: an Hybridization Approach of Metaheuristics with Column Generation" Dorabella Santos, Amaro de Sousa, Filipe Alvelos and Michał Pióro show a hybridization approach using a metaheuristic with column generation not only at the beginning but also during the space search.

Noriaki Kamiyama, Tatsuya Mori, Ryoichi Kawahara, Shigeaki Harada and Haruhisa Hasegawa in "Analyzing Influence of Network Topology on Designing ISP-operated CDN" investigate the properties of the topological locations of nodes at which cache placement is effective; they classify the evaluated networks into two types and determine the optimum cache count in each network type. In "Least Cost Routing (LCR) solution for inter-domain traffic distribution", Miroslaw Kantor, Piotr Chołda and Andrzej Jajszczyk consider the problem of route selection in a multi-homed stub network to optimize transit costs and paths performance offered by an operator to its customers is addressed.

Hiroshi Yamada, Takeshi Yada and Hiroto Nomura in "Developing network configuration management database system and its application" show the work in progress to develop their database system and several data federation methodologies for network management. "Proactive recovery from multiple failures utilizing overlay networking technique" by Go Hasegawa, Takuro Horie and Masayuki Murata proposes a proactive recovery method against multiple network failures for large-scale packet switching networks that exploits the overlay networking technique. Tomasz Mrugalski, Jozef Wozniak and Krzysztof Nowicki in "Dynamic Host Configuration Protocol for IPv6 Improvements for Mobile Nodes" propose several new mechanisms that enable faster IPv6 reconfiguration including a priori knowledge about configuration available at destination locations.
"Lightpath Design \& Management System for IP-overCWDM Networks with ROADMs, Employing Parallel Processing" by Raja Zahilah, Yuta Ogino, Soichiro Nishiuma, Md. Nooruzzaman, Nguyen Thi Thanh Thuy, Osanori Koyama and Yutaka Katsuyama provides information on the authors' system that can process applications composed of some sub applications in parallel in multi machines for highspeed performance in IP-over-CWDM networks. Krzysztof Walkowiak and Jacek Rak in "Simultaneous Optimization of Unicast and Anycast Flows and Replica Location in Survivable Optical Networks" formulate new mixed-integer programming models to find the optimal paths for anycast and unicast connections, as well as to find optimal locations for the of replica servers.

Jean-Philippe Joseph in "PSTN Services Migration to IMS-Are Service Providers Finally Reaching the Tipping Point for Large Scale Migrations?" examines the SP's needs, the services architectures and platforms, the access technology alternatives, the inherent PSTN interworking requirements that need to be supported during the transition, and pending implementation challenges. The paper "Integrated optimization of aggregation and core for varying NGN architectures" by Ulrich Menne, Roland Wessäly, Maren Martens and Andreas Bley presents an optimization framework to support network operators in assessing central strategic design and architecture questions regarding their next generation networks.

Kurt Majewski and Michael Koonert in "Analytic uplink cell load approximation for planning fractional power control in LTE networks" establish a system of cell equations for the uplink cell loads and blocking probabilities, taking Fractional Power Control parameters, irregular cell areas, service specific user distributions, and mutual cell interference into account. In "Analytical model of the multi-service cellular network servicing multicast connections" by Damian Parniewicz, Maciej Stasiak and Piotr Zwierzykowski, a new analytical method of blocking probability calculation in multi-service cellular systems with multicast connections is proposed.

Bartosz Jankowski, Wojciech Mazurczyk and Krzysztof Szczypiorski in "PadSteg: Introducing Inter-Protocol Steganography" introduce a new steganographic system called PadSteg (Padding Steganography) that represents interprotocol steganography, i.e., usage of relation between two or more protocols from the TCP/IP stack to enable secret communication. Wojciech Mazurczyk, Miłosz Smolarczyk and Krzysztof Szczypiorski in "On Information Hiding in Retransmissions" present an idea and experimental results for RSTEG (Retransmission Steganography), which is an intra-protocol hybrid network steganography method that enables hidden communication by not acknowledging a suc- 
cessfully received packet in order to intentionally invoke retransmission.

Finally, in the last paper in this issue entitled "Quantifying CAPEX Savings of Homing Architectures Enabled by Future Optical Network Equipment" by Eleni Palkopoulou, Dominic Schupke and Thomas Bauschert focuses on savings with respect to network equipment capital expenditures (CAPEX) achieved by transitioning to different homing architectures in an IP-over-optical data unit setting.

We believe that this Special Issue will contribute to enhancing knowledge in many diverse areas of the modern telecommunications. In addition, we also hope that the presented results will stimulate further research in the important areas of this scientific field.

Open Access This article is distributed under the terms of the Creative Commons Attribution Noncommercial License which permits any noncommercial use, distribution, and reproduction in any medium, provided the original author(s) and source are credited.

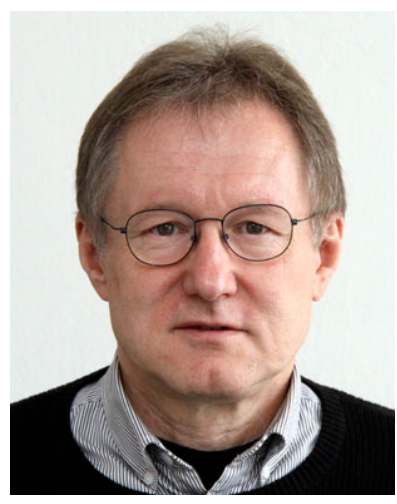

Michał Pióro is a full professor and the head of the Computer Networks and Switching Division in the Institute of Telecommunications at the Warsaw University of Technology, Poland, and a full professor at Lund University, Sweden. He received the Ph.D. degree in telecommunications in 1979 and the D.Sc. degree in 1990, both from the Warsaw University of Technology. In 2002 he received a Polish State Professorship. His research interests concentrate on modeling, design and performance evaluation of telecommunication networks. He is an author of four books and around 150 technical papers presented in the telecommunication and OR journals and conference proceedings. He has lead many national and international research projects for telecom industry and $\mathrm{EC}$ in the field.

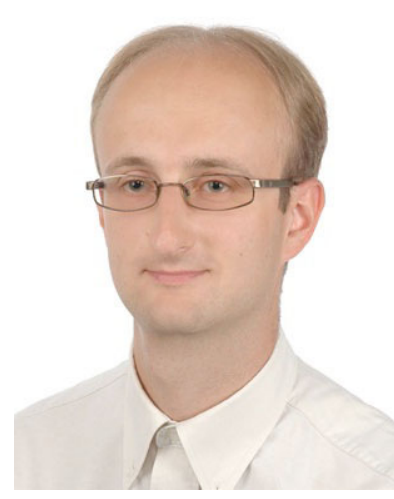

Jacek Rak holds M.Sc. and Ph.D. degrees in computer science (options: computer networks, and computer communications, accordingly) received with distinction in 2003 and 2009, respectively from Gdansk University of Technology (GUT), Poland. He is currently an assistant professor at the Department of Computer Communications of the Faculty of Electronics, Telecommunications and Informatics at GUT. His main research areas include: routing, design, dimensioning and analysis of high-speed wavelength routed backbone networks with focus on survivability. Dr Rak was involved in many projects related to optimization of reliable computer networks. He was the TPC Co-Chair of NETWORKS 2010 Conference, Publication Chair of NETWORKS 2010 and BCFIC 2011 conferences. He also served as a TPC member/ reviewer of important conferences on communications, e.g. IEEE ICC, IEEE GLOBECOM, DRCN, and journals, e.g. IEEE/ACM Transactions on Networking, or IEEE Communications Letters. Dr Rak is a member of IEEE (and IEEE Communications Society), and IFIP TC6 WG 6.10 (Photonic Networking Group). He is also the founder and the General Chair of the International Workshop on Reliable Networks Design and Modeling (RNDM).

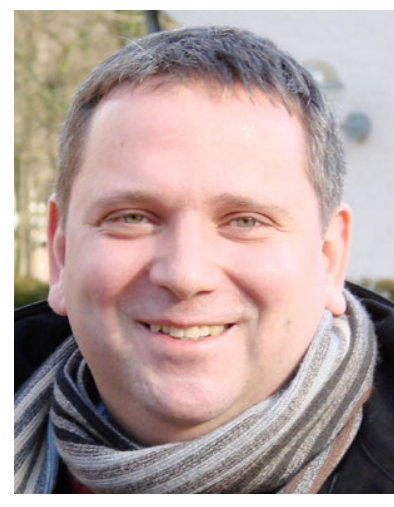

Krzysztof Szczypiorski holds an M.Sc. (1997) and a Ph.D. (2007) in telecommunications both with honours from the Faculty of Electronics and Information Technology, Warsaw University of Technology (WUT), and is an Assistant Professor at WUT. He is the founder and head of the International Telecommunication Union Internet Training Centre (ITU-ITC), established in 2003. He is also a research leader of the Network Security Group at WUT (secgroup.pl). His research interests include network security, steganography and wireless networks. He is the author or co-author of over 140 publications including 90 papers, two patent applications, and 40 invited talks. 